\title{
O Papel do Conselho de Administração na Contratação da Empresa de Auditoria: Evidências Empíricas no Brasil
}

\begin{abstract}
Resumo
O propósito deste trabalho foi investigar se existe relação entre as características do conselho de administração e o tipo de firma de auditoria externa independente contratada em companhias abertas listadas na Bolsa de Valores de São Paulo (BM\&FBovespa). Foram consideradas três características do conselho de administração: independência, duplicidade de cargo e tamanho. Já para as firmas de auditoria, foram consideradas duas características: tamanho e nível de especialização no segmento de atuação da companhia, baseados em duas medidas (estratégia de mercado e participação de mercado). Os dados foram coletados do website da Comissão de Valores Mobiliários (CVM) e da Economática, compreendendo os períodos de 1998 a 2006. Os resultados indicam que a duplicidade de cargo está associada à contratação de firmas de auditoria maiores (BigN) e especialistas. Tais resultados contribuem para a discussão sobre como a estrutura de governança corporativa influencia a contratação do auditor, ampliando o entendimento do papel do conselho de administração no monitoramento e controle das companhias abertas brasileiras.
\end{abstract}

Palavras-chave: Governança Corporativa; Auditoria; Conselho de Administração.

\section{Kadija Celante Pizetta}

Mestre em Administração de Empresas (Fucape Business School), Analista Financeira da Ivista Consultoria e Estratégia Imobiliária Ltda. Contato: Avenida Nossa Senhora dos Navegantes, 955, Enseada do Suá, Vitória, ES, CEP 29050-335.

E-mail: kadija.celante@hotmail.com

\section{Fábio Moraes da Costa}

Doutor em Ciências Contábeis (USP), Professor da Fucape Business School. Contato: Av. Fernando Ferrari, 1358, Boa Vista, Vitória, ES, CEP: 29075-505.

E-mail: fabio@fucape.br 


\section{Introdução}

O objetivo desta pesquisa foi investigar se as características do conselho de administração estão relacionadas com a contratação do tipo de firma de auditoria externa independente.

A teoria da agência sugere que o conselho de administração é um mecanismo usado para monitorar as ações dos gestores em favor do principal (Jensen \& Meckling, 1976). Além disso, também faz parte da estrutura de governança corporativa a contratação de uma firma de auditoria independente.

Fama e Jensen (1983) já afirmavam que as características do conselho de administração interferem na forma de monitoramento. Nesse sentido, dado que as características do conselho de administração interferem na forma de monitoramento (Fama \& Jensen, 1983), então poderia haver influência direta na contratação do tipo de auditoria externa independente.

Os investidores contratam auditores externos com o objetivo de diminuir a margem do gestor para efetuar determinadas escolhas contábeis para benefício próprio (Watts \& Zimmerman,1986). DeFond (1992) define que a qualidade no serviço de auditoria externa consiste na probabilidade de um auditor detectar e divulgar irregularidades e distorções em demonstrações contábeis. Assim, autores como Solomon, Shields e Whittington (1999) afirmam que, devido ao conhecimento mais profundo e a experiência no setor de atuação, os auditores externos especializados fazem análises contábeis mais precisas.

Beasley e Petroni (2001) e Lin e Liu (2009) estudaram a relação entre o conselho de administração e a contratação da auditoria externa especializada, apresentando evidências que relacionam as características do conselho (independência do conselho, separação de cargo e tamanho) e a contratação de auditores com expertise no setor da companhia.

O Brasil apresenta características diferenciadas dos mercados onde trabalhos similares foram desenvolvidos. A Comissão de Valores Mobiliários (CVM) instituiu o rodízio obrigatório de auditoria externa por meio da Instrução CVM 308 (1999). A troca deveria acontecer, no máximo, até 2004, mas houve antecipação obrigatória para o caso das companhias auditadas pela Arthur Andersen, em 2002. Assim, as especificidades do contexto brasileiro podem levar a resultados diferenciados da literatura internacional.

A metodologia empregada consistiu na aplicação de umas regressões logísticas e regressões multinomiais. Os resultados encontrados indicam que somente a duplicidade de cargos, em que o CEO e o Chairman of the Board são pessoas diferentes, acaba influenciando a contratação de firmas de auditoria maiores - BigN (ao longo do período analisado, a quantidade de empresas consideradas "grandes" (Big) foi alterada de 5 para 4. Assim, foi utilizada a denominação "BigN") e especializadas no segmento de atuação da empresa. Assim, apenas uma das três características do conselho de administração aparenta influenciar o processo de contratação dos auditores.

\section{Referencial Teórico}

\subsection{Conflitos de Interesses}

Os conflitos de interesses originam-se do desalinhamento de incentivos entre principal e agente (Jensen \& Meckling, 1976), levando fornecedores de capital a buscarem, ex-ante, proteção via estabelecimento de contratos. Porém, como eles são imperfeitos e incompletos, contingências e eventos inesperados não estão contemplados em suas cláusulas (Zingales, 1998). Como consequência, os gestores continuariam com determinadas flexibilidades que levariam à potencial expropriação do principal (Shleifer \& Vihny, 1997).

A assimetria informacional entre gestores e proprietários, aliada a contratos incompletos, pode levar ao comportamento oportunístico dos primeiros (Tirole, 2001). Nesse sentido, o principal busca o alinhamento de interesses por meio da implementação de mecanismos de governança corporativa (Shleifer \& Vishny, 1997). 
A proteção aos investidores torna-se relevante, pois há, em muitos países, a expropriação de acionistas minoritários e de credores por parte dos acionistas controladores (La Porta, Lopes-de-Silanez, Sheifer \& Vishny, 2000). Segundo DeFond (1992) e Beasley e Petroni (2001), políticas de controle acionário, composição do conselho de administração e auditoria externa são mecanismos de governança corporativa mais utilizados para alinhamento de interesses.

O controle acionário pode ser motivo de conflitos de agência, tanto para situações de alta concentração quanto para alta dispersão. Anderson (1999) apresenta evidências sobre a alta concentração acionária no Brasil: os três maiores acionistas possuíam, em média, $74 \%$ das ações ordinárias e $49 \%$ de todas as ações. Nas empresas que apresentam características de concentração acionária, o gestor-proprietário tem maior conhecimento sobre os assuntos corporativos, tornando mais fácil a expropriação do acionista minoritário (Fama \& Jensen, 1983). Por outro lado, empresas com controle acionário muito disperso, sendo controladas por gestores, apresentam margem para o gestor manipular os resultados, desviando recursos dos acionistas da empresa (Dey, 2008).

Outro mecanismo comum de governança corporativa consiste no conselho de administração e sua estrutura: independência, duplicidade de cargo (quando a mesma pessoa ocupa o cargo de CEO (Chief executive officer) e de Chairman of the Board (Presidente do Conselho de Administração)) e tamanho (Becht, Bolton \& Roell, 2002). Fama e Jensen (1983) atribuem ao conselho de administração a responsabilidade de aprovar os projetos da empresa e monitorar sua execução. Para Becht et al. (2002), o conselho de administração tem a missão de contratar o CEO, monitorá-lo e votar em decisões importantes, como fusões e aquisições, mudanças na forma de remuneração e na estrutura de capital da empresa.

Existem três formas de buscar garantir a integridade do conselho de administração muito difundida entre os pesquisadores. A primeira é a presença de conselheiros independentes na composição do conselho, pois teriam menor propensão a serem manipulados e, adicionalmente, diminuiriam a liberdade de ações dos gestores (Beasley \& Petroni, 2001) (2001). Assim, conselheiros independentes são definidos como membros livres de qualquer ligação com a direção da empresa e o acionista majoritário (Becht et al., 2002).

A segunda forma é a separação do cargo de CEO e Chairman, pois diminui o poder do CEO, aumentando a capacidade de monitoramento da gestão da empresa (Fama \& Jensen, 1983). Já a terceira forma consiste no tamanho do conselho de administração, pois interfere na confiança e agilidade das decisões tomadas. Tanto conselhos com poucos membros quanto com muitos participantes podem levar ao conluio ou à dificuldade de tomada de decisão, respectivamente (Bhuiyan, Roudaki \& Clark, 2010).

A associação entre as práticas de governança corporativa e contratação de auditorias especializadas foi estudada por autores como Watts e Zimmerman (1986), DeFond (1992), Craswell, Francis e Taylor (1995), Beasley e Petroni (2001) e Lin e Liu (2009). Eles concluem que a união desses mecanismos reduz o conflito de agência, resultando em melhor desempenho e maior probabilidade de o auditor detectar e divulgar violações no sistema contábil da empresa.

\subsection{Contratação da Empresa de Auditoria}

Jensen e Meckling (1976) sugerem que a demanda por auditoria tem origem no desejo de reduzir o gerenciamento das informações prestadas aos fornecedores de capital. Assim, a auditoria tem sido constantemente utilizada como um dos mecanismos de redução da assimetria de informação e de monitoramento entre provedores de fundos e gestores responsáveis por administrar esses recursos (Watts e Zimmerman, 1986).

Segundo DeFond (1992), a auditoria externa é uma forma de sinalizar para os investidores maior nível de governança corporativa, o que resulta em maior segurança para o mercado.

Como os conflitos de interesse também são aplicáveis às próprias firmas de auditoria (Antle, 1982), empresas que não possuam uma estrutura de governança adequada poderiam expropriar acionistas minoritários por meio da contratação de firmas de auditoria menos independentes e/ou menos competen- 
tes (Beasley \& Petroni, 2001; Lin \& Liu, 2009). Este seria o caso do conselho de administração, que, geralmente, é responsável pela contratação do auditor.

A qualidade dos serviços prestados pelos auditores depende da habilidade de detecção de distorções e de informar tais distorções às partes interessadas (De Angelo, 1981). Assim, o resultado da auditoria dependeria da interação entre o nível de independência em relação ao cliente e à competência para execução do trabalho (DeFond, 1982).

Existem alguns tipos de conhecimento que parecem ser determinantes para expertise do auditor. O primeiro é o conhecimento de domínio geral, que é adquirido por meio da formação profissional e experiência. O segundo está relacionado com especialização em determinado setor e conhecimento adquirido na prestação de serviço para clientes ou indústria específica. O terceiro tipo de conhecimento é o do negócio em geral, o qual se obtém por meio de instrução formal. Por último, é necessário que o auditor tenha habilidade para resolver problemas, que inclui reconhecer as relações, interpretar dados e ter bom senso (Bonner \& Lewis, 1990).

Resultados empíricos indicam que algumas características das firmas de auditoria estariam positivamente relacionadas com serviços de melhor qualidade, como maior propensão à emissão de relatórios modificados ou menor nível de gerenciamento de resultados (vide, por exemplo, Gramlin \& Stone, 2001). O nível de especialização e o tamanho da firma de auditoria são comumente apontados como características ligadas à independência e competência profissional (DeFond, 1992; Craswell, Francis \& Taylor, 1995; Francis, 2004).

A contratação da empresa de auditoria externa especializada pode ser um mecanismo importante para reduzir conflitos de agência. Auditores externos especializados possuiriam maior grau de experiência no setor, sendo capazes de encontrar e divulgar irregularidades e distorções nas demonstrações financeiras da empresa contratante (Defonf, 1992; Beasley e Petroni, 2001).

Craswell et al. (1995) utilizam duas métricas para identificar auditores especializados em determinados setores: participação de mercado (market share) e estratégia de negócio (market strategy). Solomon, Shields e Whittington (1999) concluem que auditorias especializadas em um determinado setor são mais precisas em suas análises do que as que não possuem especialização.

Outra forma de mensuração do nível de qualidade dos auditores externos consiste em seu tamanho. Segundo Francis (2004), as auditorias consideradas "grandes", como as chamadas de BigN, vão em média oferecer qualidade superior aos seus clientes devido à independência e à reputação da marca (De Angelo, 1981; Lennox, 1999). A maior parte das evidências empíricas indica que empresas auditadas por firmas consideradas "BigN" apresentam menores níveis de accruals discricionários (Dechow, Ge \& Schrand, 2010).

\subsection{Desenvolvimento das Hipóteses}

A independência do conselho de administração está relacionada com a presença de membros externos no conselho de administração. Fama (1980) e Fama e Jensen (1983) acreditam que a presença de membros independentes é um importante mecanismo de monitoramento.

Para Beasley e Petroni (2001) e Carcello, Hermanson, Neal \& Riley (2002), conselheiros independentes contribuem para a proteção dos investidores. Dado que auditores especializados e de maior tamanho (BigN) contribuiriam para a execução de serviços de maior qualidade, a primeira hipótese levantada é apresentada a seguir:

- H1 - Companhias com conselhos de administração mais independentes têm maior probabilidade de contratarem firmas de auditoria especializadas e de maior tamanho (BigN).

A segunda característica é a duplicidade de cargo do CEO e Chairman. Quando a mesma pessoa ocupa ambas as posições, o poder de monitoramento é reduzido, pois o presidente do conselho de admi- 
nistração fica responsável pelo alto monitoramento e pode influenciar os outros membros do conselho a aprovar projetos que maximizem sua própria utilidade (Beasley, 1996; Imhoff, 2003).

A relação entre duplicidade de cargo e contratação da empresa de auditoria foi estudada por Lin e Liu (2009), que apresentam evidências empíricas que indicam que quando há duplicidade de cargo, há menor probabilidade de contratação de uma firma de auditoria especializada e maior. Nesse sentido, a segunda hipótese foi formulada:

- H2 - Companhias que não apresentam duplicidade de cargo do CEO e Chairman of the Board têm maior probabilidade de contratarem firmas de auditoria especializadas e de maior tamanho (BigN).

A terceira caracteristica é o tamanho do conselho de administração, pois pode influenciar na agilidade e capacidade de tomada de decisões. Embora Hermalin e Weisbach (1991) sugiram que o tamanho e a composição do conselho são endógenos à empresa e é pouco provável que exista um tamanho ou uma composição do conselho que seja aplicável a todos os mercados, Bhuiyan et al. (2010) argumentam que conselho de administração composto de poucos membros pode não ter visão geral do negócio. Por outro lado, com muitos membros, pode-se ter dificuldade de se chegar a alguma conclusão.

Fama e Jensen (1983) sugerem uma quantidade ideal para o conselho de administração entre 7 e 8 membros. Já o Instituto Brasileiro de Governança Corporativa (IBGC, 2009) sugere uma quantidade ótima entre 5 e 9 membros para compor o conselho de administração no Brasil. Lin e Liu (2009), em um estudo realizado na China, concluem que empresas com maior número de conselheiros apresentam maior probabilidade de contratação de firmas de auditoria especializadas, resultando em melhor monitoramento e supervisão das ações dos gestores. Assim, a terceira hipótese foi desenvolvida:

- H3 - Companhias com conselhos de administração maiores têm maior probabilidade de contratarem firmas de auditoria especializadas e de maior tamanho (BigN).

A análise em conjunto das três hipóteses permitirá avaliar se as características do conselho de administração estão associadas à contratação da auditoria externa especializada e maior tamanho (BigN), tema central desta pesquisa.

\section{Metodologia}

Os dados foram obtidos por meio de consulta aos websites das companhias, da Comissão de Valores Mobiliários e a base de dados Economática. As informações sobre governança corporativa foram obtidas da mesma base e já foi utilizada por Lopes e Walker (2012) para construção do BCGI (Brazilian Corporate Governance Index). Já a base de auditoria foi utilizada por Pereira (2011). O software Economática também foi utilizado para captação das variáveis contábeis.

Os dados das companhias abertas listadas na BM\&FBovespa foram coletados para os períodos de 1998, 2000, 2002, 2004 e 2006. O motivo para utilização desses respectivos anos é a disponibilidade da base de dados existentes na base do BCGI. A amostra final é composta de 1.393 observações, sendo que 273 são referentes ao ano de 1998; 302, a 2000; 301, a 2002; 334, a 2004; e 183 são referentes a 2006. Sabe-se que, como as variáveis foram computadas a cada dois anos, tal fato pode interferir nos resultados e deve ser levado em consideração nas análises e generalizações. Ressalta-se, porém, que as estruturas de governança não tendem a se alterar, significativamente, em curtos espaços de tempo.

A técnica estatística aplicada para análise dos dados é a regressão logística binária e multinomial. A regressão logística binária é interpretada em termos de razão de chances (odds ratio - OR), a qual considera a chance de obter um resultado negativo (entre 0 e 1 ) e um resultado positivo (entre 1 a $\infty$ ). A "chance" é a probabilidade da ocorrência de um evento dividida pela probabilidade de não ocorrência do mesmo evento. 
A interpretação da regressão logística multinomial é similar, porém, utiliza-se a Razão de Risco Relativo (RRR), a qual considera a probabilidade do evento ocorrer no grupo de controle versus outros grupos analisados. A RRR indica, para uma mudança de categoria de uma variável dependente (mantida as demais sem mudanças), maior ou menor probabilidade de associação de uma dada categoria da variável independente, em comparação ao grupo de controle (também conhecido como grupo de comparação).

\subsection{Proxies Empíricas}

Nesta seção, serão descritas duas proxies para mensurar o tipo de firma de auditoria.

\subsubsection{Proxy para Tamanho da Auditoria}

As auditorias conhecidas como BigN são consideradas especializadas por diversos pesquisadores (Beasley \& Petroni, 2001; Fan e Wong, 2005; Francis, 2004), por terem amplo conheçimento e terem uma reputação a zelar. Por isso, a primeira analise é testada com a proxy tamanho da auditoria.

Nos anos de 1998, 2000 e 2002, foram consideradas cinco empresas de auditoria Big: Arthur Andersen, Deloitte Touche Tohmatsu, Ernst \& Young, KPMG e Pricewaterhousecoopers. Nos anos de 2004 e 2006, após a extinção da Arhur Andersen, somente quatro empresas foram classificadas como Big.

\subsubsection{Proxy para Nível de Especialização}

Simunic (1980) e Solomon et al. (1999) sugerem que treinamento e especialização em uma indústria específica aumentam a eficiência da auditoria externa e a tornam especializada naquele setor.

Existem duas medidas comumente utilizadas para mensuração do nível de especialização: estratégia de mercado e participação de mercado (Gramling \& Stone, 2001).

A estratégia de mercado (market strategy) consiste na avaliação de quanto um determinado setor representa do faturamento total da firma de auditoria. Para o seu cálculo, é utilizada a razão entre o somatório das receitas dos clientes da firma de auditoria no setor em análise em relação ao somatório das receitas de todos os clientes auditados por essa firma de auditoria.

Já a participação de mercado (market share) consiste na avaliação da representatividade da firma de auditoria em um determinado setor. Para o seu cálculo, é utilizada a razão entre o somatório das receitas dos clientes da firma de auditoria no setor em análise em relação ao somatório das receitas totais de todas as companhias do setor em análise.

Craswell et al. (1995) classificam uma firma de auditoria como especializada se o resultado de pelo menos uma das métricas (estratégia de mercado ou participação de mercado) foi superior a $20 \%$. Nesta pesquisa, com o objetivo de avaliar se os resultados são sensíveis ao patamar de $20 \%$, também foi considerado o valor de $10 \%$.

\subsection{Identificação das Variáveis}

A seguir será apresentada a Tabela 1 com as variáveis utilizadas nesta pesquisa e suas descrições. São considerados dois tipos de auditoria externa: primeiro pelo tamanho, definido nessa pesquisa como Big, e o segundo, por participação e estratégia em um setor específico, que visam identificar firmas de auditoria especializadas. Devido à analise de sensibilidade e união das duas formas de mensuração do nível de especialização, a Tabela 1 apresenta cinco variáveis dependentes. 
Conforme a Tabela 1, pode-se observar que $40 \%$ das empresas analisadas optam pela não contratação de firmas de auditoria Big. Quando analisado o nível de especialização, e adotando a combinação das proxies market strategy/market share, a quantidade de empresas que optam por contratar auditoria não especialista aumenta para 54.

Tabela 1

\section{Descrição das variáveis}

\begin{tabular}{|c|c|c|c|c|c|}
\hline Variáveis dependentes & Descrição & Resposta & Frequência & Porcentagem & Total \\
\hline \multirow{2}{*}{ BIG } & \multirow{2}{*}{ Auditoria externa considerada BigN } & 0 & 557 & $40 \%$ & \multirow{2}{*}{1.393} \\
\hline & & 1 & 836 & $60 \%$ & \\
\hline \multirow{2}{*}{ ESP20\% } & \multirow{2}{*}{$\begin{array}{l}\text { Auditoria externa considerada } \\
\text { especializada com base nas proxies de } \\
\text { market share e market strategy (20\%) }\end{array}$} & 0 & 749 & $54 \%$ & \multirow{2}{*}{1.393} \\
\hline & & 1 & 644 & $46 \%$ & \\
\hline \multirow[b]{2}{*}{ ESP10\% } & \multirow{2}{*}{$\begin{array}{l}\text { Auditoria externa considerada } \\
\text { especializada com base nas proxies de } \\
\text { market share e market strategy (10\%) }\end{array}$} & 0 & 419 & $30 \%$ & \multirow{2}{*}{1,393} \\
\hline & & 1 & 974 & $70 \%$ & \\
\hline \multirow{4}{*}{ BIGESP20\% } & \multirow{4}{*}{$\begin{array}{l}\text { Auditoria externa considerada BigN e } \\
\text { especializada com base nas proxies de } \\
\text { market share e market strategy ( } 20 \%)\end{array}$} & 0 & 419 & $30 \%$ & \multirow{4}{*}{1.393} \\
\hline & & 1 & 138 & $10 \%$ & \\
\hline & & 2 & 330 & $24 \%$ & \\
\hline & & 3 & 506 & $36 \%$ & \\
\hline \multirow{4}{*}{ BIGESP10\% } & \multirow{4}{*}{$\begin{array}{l}\text { Auditoria externa considerada } \\
\text { especializada com base nas proxies de } \\
\text { market share e market strategy (10\%) }\end{array}$} & 0 & 294 & $21 \%$ & \multirow{4}{*}{1.393} \\
\hline & & 1 & 263 & $19 \%$ & \\
\hline & & 2 & 125 & $9 \%$ & \\
\hline & & 3 & 711 & $51 \%$ & \\
\hline \multicolumn{6}{|l|}{ Variáveis Independentes } \\
\hline \multirow{2}{*}{ INDEP } & \multirow{2}{*}{ Independência do conselho } & 0 & 86 & $6 \%$ & \multirow{2}{*}{1.393} \\
\hline & & 1 & 1.307 & $94 \%$ & \\
\hline \multirow{2}{*}{ DC } & \multirow{2}{*}{ Duplicidade de Cargo } & 0 & 495 & $36 \%$ & \multirow{2}{*}{1.393} \\
\hline & & 1 & 898 & $64 \%$ & \\
\hline \multirow{2}{*}{ TOT } & \multirow{2}{*}{ Tamanho do conselho } & 0 & 612 & $44 \%$ & \multirow{2}{*}{1.393} \\
\hline & & 1 & 781 & $56 \%$ & \\
\hline \multicolumn{6}{|l|}{ Variáveis de controle } \\
\hline \multirow{2}{*}{ CA } & \multirow{2}{*}{ Controle Acionário } & 0 & 975 & $70 \%$ & \multirow{2}{*}{1.393} \\
\hline & & 1 & 418 & $30 \%$ & \\
\hline \multirow{2}{*}{ EA } & \multirow{2}{*}{ Estrutura Acionária } & 0 & 1.051 & $25 \%$ & \multirow{2}{*}{1.393} \\
\hline & & 1 & 342 & $75 \%$ & \\
\hline ENDIV & Alavancagem & continua & & & 1.393 \\
\hline LNAT & Logaritimo do Ativo total & continua & & & 1.393 \\
\hline
\end{tabular}

A variável BIGESP é uma extensão do modelo e faz a combinação entre as duas proxies de auditoria externa especializada. Das empresas pesquisadas, destacam-se as empresas que optaram por contratar firmas de auditoria "não Big" e não especializadas (21\%) e aquelas que contrataram "Big" e especializadas (21\%).

Adotando o parâmetro de 10\% para definir auditoria externa especialista, pode-se observar que as porcentagens de empresas consideradas especializadas aumentam conforme esperado. 
Em relação às características do conselho de administração: a primeira é a variável INDEP, indicando que somente $6 \%$ dos conselhos de administração não seriam considerados independentes, dado que pode acabar por limitar os resultados das regressões logísticas.

A segunda variável independente, focada na duplicidade de cargos (DC), indica que a maior parte das companhias (64\%) possui pessoas diferentes ocupando os cargos de CEO e de Chairman.

A terceira variável independente, que representa o tamanho do conselho (TOT), indica relativo equilíbrio entre companhias que possuem entre 5 e 9 membros (56\%) e as que não possuem (46\%).

\subsection{Modelo de Regressão}

A equação 1, a seguir, é utilizada para verificar a associação entre característica do conselho de administração e a contratação do tipo de firma de auditoria:

$$
\mathrm{Y}_{i, t=} \beta_{0}+\beta_{1} \mathrm{INDEP}_{i, t}+\beta_{2} \mathrm{DC}_{i}+\beta_{3} \mathrm{TOT}_{i, t}+\beta_{4} \mathrm{CA}_{i, t}+\beta_{5} \mathrm{EA}_{i, t}+\beta_{6} \mathrm{ALA}_{i, t}+\beta_{7} \mathrm{AT}_{i, t}+\varepsilon_{i, t}
$$

Em que:

- $\mathrm{Y}_{i t}=$ Variáveis dependentes:

- $\quad$ BIG = Medida de Auditoria externa especializada, assumindo valor 1 para auditorias consideradas Big e caso contrário 0.

- $\quad \mathrm{ESP}=$ Medida de Auditoria externa especializada, assumindo valor 1 para auditorias consideradas especialistas, com base nas proxies de estratégia de mercado e participação de mercado, e caso contrário 0 .

- $\quad$ BIGESP = Medida de Auditoria externa especializada, determinada pela combinação de auditoria considerada BigN e especializada: assumindo valor 0 para não BigN e não especializada; 1 para não BigN e especializada; 2 para BigN e não especializada; e 3 para BigN e especializada.

- Variáveis independentes:

- $\quad$ INDEP = Conselho Independente: assume valor 1 quando a presença de insider é menor que 0,5 e 0 , caso contrário;

- $\quad \mathrm{DC}=$ Duplicidade de Cargo: quando não houver Duplicidade de Cargo, assume valor 1 e 0 caso contrário;

- TOT = Tamanho do Conselho de Administração: assume valor 1 para empresas que têm conselho formado entre 5 e 9 membros e 0 , caso contrário;

- $\quad \mathrm{CA}=$ Concentração Acionária: assume valor 1 quando acionista controlador possui menos que $50 \%$ da ações ordinárias e 0 , caso contrário;

- $\quad \mathrm{EA}=$ Estrutura Acionári: assume valor 1 quando o percentual das ações ordinárias $(\mathrm{ON})$ em relação ao total $(\mathrm{ON}+\mathrm{PN})$ é maior que $80 \%$ e 0 , caso contrário.

- $\quad \mathrm{ALA}=$ nível de endividamento;

- $\mathrm{AT}=$ Logaritmo do Ativo Total;

- $\varepsilon=$ termo de erro da regressão

\section{Resultados e Discussões}

Nesta seção são descritos e comentados os resultados encontrados nas regressões logísticas binárias e multinomiais. A regressão logística foi utilizada quando a variável dependente consistiu na classificação de firmas de auditoria com base em seu tamanho (BIG) ou especialização (ESP). Já a regressão multinomial foi utilizada quando a combinação das características da firma de auditoria (BIGESP) foi levada em consideração. 
$\mathrm{Na}$ Tabela 2, são apresentados os resultados referentes à associação das características do conselho de administração e a contratação da auditoria externa BIG. Nas Tabelas 3 e 4, são apresentados os resultados referentes à associação das características do conselho de administração e a contratação da auditoria especializada especialista (ESP). Por fim, nas tabelas 5 e 6 , são apresentados os resultados referentes à associação das características do conselho de administração e a contratação da auditoria externa baseada na combinação de seu tamanho e especialização (BIGESP).

A primeira análise realizada abordou as características do conselho de administração e a contratação da auditoria externa BigN. Na Tabela 2, a seguir, são apresentados os resultados da regressão logística binária:

Tabela 2

Regressão Logística Binária para Características do Conselho de Administração versus Contratação da Auditoria Externa BigN

\begin{tabular}{ccll}
\hline BIG & Sinal esperado & OR & $\mathbf{P}>|\mathbf{z}|$ \\
\hline INDEP & + & 0,940 & 0,813 \\
\hline DC & + & 1,401 & $0,012^{\star * *}$ \\
\hline TOT & + & 0,753 & $0,033^{* *}$ \\
\hline CA & - & 0,812 & 0,135 \\
\hline EA & - & 0,591 & $0,000^{* * *}$ \\
\hline ALA & + & 0,877 & 0,397 \\
\hline AT & + & 1,867 & $0,000^{* * *}$ \\
\hline Prob $>$ chi2 $=\mathbf{0 , 0 0 0}$ & Pseudo $\mathbf{R 2}=\mathbf{0 , 2 1 9}$ & Número de observações $=\mathbf{1 . 3 9 3}$ \\
\hline
\end{tabular}

** Estatisticamente significantes ao nível de 5\%; *** Estatisticamente significantes ao nível de 1\%.

O resultado do odds ratio $(\mathrm{OR}>1)$ de Duplicidade de Cargo $\left(\beta_{2}\right)$ mostra-se positivo ao nível de $1 \%$. Esse resultado sugere que empresas que apresentam características de separação de cargo de CEO e Chairman possuem maior probabilidade de contratarem firmas de auditoria BigN.

Já o tamanho do conselho $\left(\beta_{3}\right)$ apresenta uma relação negativa $(\mathrm{OR}<1)$, sugerindo que empresas que possuem entre 5 a 9 membros têm menor probabilidade de contratarem firmas de auditoria BigN. Esse resultado não está em linha com o esperado pela revisão de literatura. Uma possível explicação possa estar relacionada à utilização de uma variável dummy, em vez do valor absoluto da quantidade de conselheiros.

Por fim, a independência do conselho $\left(\beta_{1}\right)$ não foi estatisticamente significativa.

As variáveis de controle "estrutura acionária" $\left(\beta_{5}\right)$ e "ativo total" $\left(\beta_{7}\right)$ foram significativas, indicando uma relação negativa para a primeira e positiva para a segunda. Ou seja, quanto maior for ativo total da empresa, maior é a probabilidade de contratar auditoria BigN, o que é coerente com a literatura (por exemplo, Lennox, 1999). Já as empresas que possuem a maioria de ações emitidas como ações ordinárias $(\mathrm{ON})$ apresentam menor probabilidade de contratação de auditorias BigN. As outras variáveis de controle, como concentração acionária $\left(\beta_{4}\right)$ e alavancagem $\left(\beta_{6}\right)$ não foram estatisticamente significantes.

Em suma, há associação entre duas características do conselho de administração e a contratação da auditoria externa Big, mas uma está em sentido contrário ao esperado. É possível apenas afirmar que H1 seria corroborada em relação à duplicidade de cargos, mas não em relação ao tamanho e independência. Esse é um resultado divergente dos achados principais de Lin e Liu (2009), que afirmam que as características do conselho interferem na contratação da empresa de auditoria Big.

Na segunda análise realizada, a auditoria externa especializada é definida com base na junção das proxies de estragégia de mercado e participação de mercado, com parâmetro de $20 \%$. Os resultados são apresentados na Tabela 3. 
Tabela 3

Regressão Logística Binária para Características do Conselho de Administração versus Contratação da Auditoria Externa Especialista a 20\%

\begin{tabular}{|c|c|c|c|}
\hline ESP & Sinal esperado & OR & $P>|z|$ \\
\hline INDEP & + & 0,668 & 0,113 \\
\hline DC & + & 1,373 & $0,014 * * *$ \\
\hline TOT & + & 1,132 & 0,315 \\
\hline CA & - & 1,014 & 0,912 \\
\hline EA & - & 0,825 & 0,176 \\
\hline ALA & + & 0,932 & 0,607 \\
\hline AT & + & 1,645 & $0,000 * * *$ \\
\hline Prob $>$ chi2 $=0,000$ & Pseudo R2 = 0,161 & Núm & 1.393 \\
\hline
\end{tabular}

** Estatisticamente significantes ao nível de 5\%; *** Estatisticamente significantes ao nível de $1 \%$.

Os resultados demonstram que separação de cargo continua sendo estatisticamente significante ao nível de $1 \%$. O OR $>1$ indica que conselhos com características de separação de cargo de CEO e chairman possuem maior probabilidade de contratar auditoria externa com participação de mercado e estratégia de negócio em seu setor de atuação. Porém, as demais características do conselho de administração não se mostraram significativas.

Conforme discutido anteriormente, para Craswell et al. (1995), dado que o percentual utilizado para definição da firma de auditoria especializada pode ser interpretado como arbitrário (20\%), torna-se necessária uma análise de sensibilidade. Assim, com base no parâmetro de 10\%, as regressões foram novamente rodadas e seus resultados são apresentados na Tabela 4, a seguir:

Tabela 4

Regressão Logística Binária para Características do Conselho de Administração versus Contratação da Auditoria Externa Especialista a 10\%

\begin{tabular}{cccc}
\hline ESP & Sinal esperado & OR & $\mathbf{P}>|\mathbf{z}|$ \\
\hline INDEP & + & 0,834 & 0,502 \\
\hline DC & + & 1,216 & 0,162 \\
\hline TOT & + & 1,010 & 0,938 \\
\hline CA & - & 1,044 & 0,768 \\
\hline EA & - & 0,624 & $0,002^{\star * *}$ \\
\hline ALA & + & 0,979 & 0,734 \\
\hline AT & + & 1,848 & $0,000^{* * *}$ \\
\hline Prob $>$ chi2 $=\mathbf{0 , 0 0 0}$ & Pseudo $\mathbf{R 2}=\mathbf{0 , 2 1 0}$ & Número de observações $=\mathbf{1 . 3 9 3}$ \\
\hline
\end{tabular}

** Estatisticamente significantes ao nível de 5\%; *** Estatisticamente significantes ao nível de 1\%.

Os resultados da Tabela 4 confirmam a previsão de que o parâmetro acaba definindo os resultados da regressão. Nessa nova análise, nenhuma das características do conselho de administração foi significativa. Nesse sentido, recomenda-se cautela na interpretação dos resultados, não permitindo afirmar que a hipótese $\mathrm{H} 2$ foi aceita, dado que é dependente do patamar utilizado para classificação das firmas de auditoria.

Na Tabela 5, são apresentados os resultados da regressão logística multinomial para a combinação entre empresas de auditoria consideradas Big e especializadas (BIGESP), com parâmetro de $20 \%$. Foram criados quatro tipos de combinação: (0) não Big e não especializada; (1) não Big e especializada; (2) Big e não especializada; e (3) Big e especializada. O grupo de comparação adotado nessa pesquisa será o grupo de auditoria externa não big e não especializada (0). Os resultados são apresentados a seguir: 
Tabela 5

\section{Regressão Logística Multinomial para Características do Conselho de Administração versus Contratação da Auditoria Externa BigN e Especialista A 20\%}

\begin{tabular}{|c|c|c|c|c|c|c|c|c|c|c|c|}
\hline \multicolumn{12}{|c|}{ Grupo de comparação = NBIGNESP - grupo 0} \\
\hline \multicolumn{4}{|c|}{ NBIGESP - Grupo 1} & \multicolumn{4}{|c|}{ BIGNESP - Grupo 2} & \multicolumn{4}{|c|}{ BIGESP - Grupo 3} \\
\hline & Sinal & RRR & $P>|z|$ & & Sinal & RRR & $P>|z|$ & & Sinal & RRR & $P>|z|$ \\
\hline INDEP & + & 0,705 & 0,366 & INDEP & + & 1,058 & 0,867 & INDEP & + & 0,662 & 0,219 \\
\hline DC & + & 1,140 & 0,538 & DC & + & 1,229 & 0,217 & DC & + & 1,783 & $0,001 * * *$ \\
\hline TOT & + & 1,518 & $0,055^{* *}$ & TOT & + & 0,835 & 0,279 & TOT & + & 0,862 & 0,380 \\
\hline CA & - & 1,309 & 0,210 & $C A$ & - & 0,919 & 0,634 & $C A$ & - & 0,851 & 0,370 \\
\hline EA & - & 0,866 & 0,531 & EA & - & 0,590 & $0.005^{* * *}$ & EA & - & 0,531 & $0,001 * * *$ \\
\hline ALA & + & 1,001 & 0,982 & ALA & + & 0,963 & 0,735 & ALA & + & 0,515 & 0,240 \\
\hline AT & + & 1,650 & $0,000 * * *$ & AT & + & 1,826 & $0.000 * * *$ & AT & + & 2,668 & $0,000 * * *$ \\
\hline \multicolumn{4}{|c|}{ Prob $>$ chi2 $=0,000$} & \multicolumn{4}{|c|}{ Pseudo R2 = 0,163 } & \multicolumn{4}{|c|}{ Numero de observações = 1.393} \\
\hline
\end{tabular}

** Estatisticamente significantes ao nível de 5\%; *** Estatisticamente significantes ao nível de 1\%.

Os resultados para o grupo 1 indicam que conselhos de administração compostos entre 5 a 9 membros possuem maior probabilidade de contratarem auditoria externa não Big e especializada. As outras características do conselho não são significativas.

Já para o grupo 2, nenhuma das três características do conselho foi estatisticamente significativa. Por fim, para o grupo 3, os resultados indicam que a companhias com separação de cargo (DC) possuem maior probabilidade de contratação de firmas de auditoria Big e especializadas.

A seguir, na Tabela 6, são apresentados os resultados, levando-se em consideração os mesmos grupos, mas com base no parâmetro de $10 \%$ para definição da firma de auditoria especializada.

Tabela 6

Regressão Logística Multinomial para Características do Conselho de Administração versus Contratação da Auditoria Externa BigN e Especialista a 10\%

\begin{tabular}{|c|c|c|c|c|c|c|c|c|c|c|c|}
\hline \multicolumn{12}{|c|}{ Grupo de comparação = NBIGNESP - grupo 0} \\
\hline \multicolumn{4}{|c|}{ NBIGESP - Grupo 1} & \multicolumn{4}{|c|}{ BIGNESP - Grupo 2} & \multicolumn{4}{|c|}{ BIGESP - Grupo 3} \\
\hline & Sinal & RRR & $P>|z|$ & & Sinal & RRR & $P>|z|$ & & Sinal & RRR & $P>|z|$ \\
\hline INDEP & + & 1,136 & 0,715 & INDEP & + & 1,749 & 0,282 & INDEP & + & 0,852 & 0,632 \\
\hline DC & + & 0,849 & 0,384 & DC & + & 0,870 & 0,548 & DC & + & 1,463 & $0,036 * *$ \\
\hline TOT & + & 1,324 & 0,137 & TOT & + & 1,020 & 0,930 & TOT & + & 0,830 & 0,300 \\
\hline CA & - & 1,106 & 0,604 & CA & - & 0,814 & 0,409 & CA & - & 0,861 & 0,426 \\
\hline EA & - & 0,696 & 0,075 & EA & - & 0,645 & 0,087 & EA & - & 0,435 & $0,000 * * *$ \\
\hline ALA & + & 0,975 & 0,737 & ALA & + & 0,525 & 0,246 & ALA & + & 0,927 & 0,609 \\
\hline AT & + & 1,719 & $0,000 * * *$ & AT & + & 1,826 & $0,000 * * *$ & AT & + & 2,817 & $0,000 * * *$ \\
\hline \multicolumn{4}{|c|}{ Prob $>$ chi2 $=0,000$} & \multicolumn{4}{|c|}{ Pseudo R2 = 0,181 } & \multicolumn{4}{|c|}{ Numero de observações = 1.393} \\
\hline
\end{tabular}

** Estatisticamente significantes ao nível de 5\%; *** Estatisticamente significantes ao nível de 1\%.

Os resultados indicam que nenhuma das 3 características do conselho de administração foi significativa para os grupos 1 e 2. Já para o grupo 3, o resultado é similar ao apresentado com o parâmetro de $20 \%$, indicando que a duplicidade de cargo é relevante para a contratação de firmas de auditoria Big e especializada. Em conjunto, os resultados corroboram parcialmente a hipótese $\mathrm{H} 3$. 
A seguir, na Tabela 7, é apresentado um resumo dos resultados encontrados nas regressões. Os sinais “+” e“-” indicam o sentido da relação (positivo ou negativo). Ao lado, é apresentada significância estatística.

Tabela 7

Resumo dos Resultados

\begin{tabular}{|c|c|c|c|c|c|c|c|c|c|c|c|c|c|c|c|c|c|c|}
\hline & \multirow{2}{*}{\multicolumn{2}{|c|}{ BIG }} & \multirow{2}{*}{\multicolumn{2}{|c|}{ ESP $20 \%$}} & \multirow{2}{*}{\multicolumn{2}{|c|}{ ESP $10 \%$}} & \multicolumn{6}{|c|}{ BIGESP 20\% } & \multicolumn{6}{|c|}{ BIGESP 10\% } \\
\hline & & & & & & & \multicolumn{6}{|c|}{ Grupo } & \multicolumn{6}{|c|}{ Grupo } \\
\hline & Sinal & $\%$ & Sinal & $\%$ & Sinal & $\%$ & 1 & $\%$ & 2 & $\%$ & 3 & $\%$ & 1 & $\%$ & 2 & $\%$ & 3 & $\%$ \\
\hline \multicolumn{19}{|c|}{ INDEP } \\
\hline DC & + & $1 \%$ & + & $1 \%$ & & & & & & & + & $1 \%$ & & & & & + & $5 \%$ \\
\hline TOT & - & $5 \%$ & & & & & + & $5 \%$ & & & & & & & & & & \\
\hline \multicolumn{19}{|l|}{ CA } \\
\hline EA & - & $1 \%$ & & & - & $1 \%$ & & & - & $1 \%$ & - & $1 \%$ & & & & & - & $1 \%$ \\
\hline \multicolumn{19}{|l|}{ ALA } \\
\hline AT & + & $1 \%$ & + & $1 \%$ & + & $1 \%$ & + & $1 \%$ & + & $1 \%$ & + & $1 \%$ & + & $1 \%$ & + & $1 \%$ & + & $1 \%$ \\
\hline
\end{tabular}

Percebe-se que a independência do conselho não foi significativa, fato que diverge dos resultados de Beasley e Petroni (2001). Porém, a explicação pode residir no fato de que quase a totalidade da amostra foi classificada como "independente" (96\%), conforme apresentado nas estatísticas descritivas.

A duplicidade de cargo possui associação positiva com a contratação de auditoria externa Big e especializada, separadamente. O mesmo resultado acontece com a combinação de BigN e especializada, sugerindo que conselhos de administração que possuem pessoas diferentes ocupando o cargo de CEO e Chairman acabam levando à contratação de firmas maiores e com conhecimentos mais específicos sobre o setor. O resultado corrobora os achados de Lin e Liu (2009), sugerindo que conselhos de administração que possuem pessoas diferentes ocupando o cargo de CEO e Chairman adotarão atitudes que intensificam o monitoramento do gestor.

A variável de controle estrutura acionária apresenta associação negativa, estando em linha com DeFond (1992). Outra variável de controle que confirma as pesquisas de DeFond (1992), Beasley e Petroni (2001) e de Lin e Liu (2009) é o tamanho da companhia, mensurado por meio de seu ativo total. Companhias maiores tendem a contratar auditores BigN e especializados.

Já as variáveis de controle "concentração acionária" e "alavancagem” não foram significativas, não corroborando pesquisas anteriores (DeFond, 1992; Petronni e Beasley, 1996). As características peculiares do mercado brasileiro podem explicar tais resultados, como a alta concentração acionária.

Por fim, conforme previsto por Craswell et al. (1995), os resultados são sensíveis ao parâmetro usado para especificar auditoria especializada.

Em suma, as evidências indicam que apenas uma característica, a duplicidade de cargos, é apresentada de maneira consistente como mecanismo que está relacionado à contratação de tipos específicos de firmas de auditoria (BigN e especializada).

\section{Considerações Finais}

O presente estudo investigou a associação entre características do conselho de administração e a contratação do tipo de firma de auditoria nas companhias listadas na BM\&FBovespa. Foram estudadas três características do conselho: independência, tamanho e duplicidade de cargo.

Os resultados encontrados evidenciam a associação entre as características do conselho de separação de cargo do CEO e Chairman e a contratação da auditoria tanto nas análises segregadas (BigN e especializada) quanto na combinação das duas métricas. Assim, pesquisas sobre governança que levem em consideração o tipo de auditor deveriam levar em consideração a variável DC. 
Os resultados contribuem para a avaliação de quais mecanismos de governança corporativa são relevantes para a tomada de decisão em contextos específicos. A duplicidade de cargo aparenta ser o motivador da contratação de firmas de auditoria com maior reputação e especializadas no segmento de atuação da companhia.

Para pesquisas futuras, sugere-se a triangulação entre as características do conselho de administração, o tipo de auditor contratado e a qualidade da informação contábil divulgada.

\section{Referências}

Antle, R. (1982). Auditor as an Economic Agent. Journal of Accounting Research, 20(2), p. 502-527.

Beasley, M, S. \& Petroni, K. (2001). Board independence and audit-firm type. Auditing: A Journal of Practice \& Theory, 20(1), p. 97-114.

Beasley, M. S. (1996). An empirical analysis of the relation between the board of director composition and financial statement fraud. The Accounting Review, 71(4), p. 443-465.

Becht, M., Bolton, P. \& Röell, A. A. (2011). Corporate Governance and Control. Finance Working Paper. n. 2, 2002. Recuperado em 26 maio, $2012 \mathrm{de}<\mathrm{http}: / /$ ssrn.com/abstract $=343461>$.

Bhuiyan, U, B., Roudaki, J. \& CLARK, M. (2010). Characteristics of the Board of Directors and Company Performance - New Zealand Evidence. Journal of Accounting - Business \& Management, 17(2), p. 49-61.

Bonner, S. \& Lewis, B. (1990). Determinants of Auditor Expertise. Journal of Accounting Research, 28(Suplemento), p. 1-20.

Carcello, V, J., Hermanson, R D., NEAL, L T. \& Riley, A, R. (2002). Board characteristics and audit fees. Contemporary Accounting Research, 19(3), p. 365-84.

Craswell, A, T., Francis, J, R. \& TAYLOR, L,S. (1995). Auditor brand name reputations and industry specializations. Journal of Accounting and Economics, 20(3), p. 297-322.

DeAngelo, L.E. (1981). Auditor size and audit quality. Journal of Accounting and Economic, 3(3), p. 183-199.

Dechow, P.M.; Ge, W. \& Schrand, C.M. Understanding Earnings Quality: A Review of the Proxies, Their Determinants and Their Consequences. Working Paper SSRN. Recuperado em 26 maio, 2011 de http://papers.ssrn.com/sol3/papers.cfm?abstract_id=1485858.

DeFond, M, L. (1992). The association between changes in client firm agency costs and auditor switching. Auditing: A Journal of Practice and Theor, 11(Spring), p. 16-31.

Dey, A. (2008). Corporate governance and Agency Conflicts. Journal of Accounting Research, 46(5), p. 1143-1181.

Fama, E, F. (1980). Agency Problems and the Theory of the Firm. The Journal of Political Economy, 88(2), p. 288-307.

Fama, E. \& Jensen, M. (1983). Separation of ownership and control. Journal of Law and Economics, 26(2), p. 301-325.

Fan, P, H, J. \& Wong, T, J. (2005). Do external auditors perform a Corporate Governance role in emerging markets? Evidence from East Asia. Journal of Accounting Research, 43(1), p. 35-72.

Francis, J. R. (2004). What do we know about audit quality? The British Accounting Review, 36(4), p. 345-368.

Gramling, A, A. \& Stone, N. D. (2001). Audit firm industry expertise: A review and synthesis of the archival literature. Journal of Accounting Literature, 20(3), p. 1-29.

Hermalin, B. E \& Weisbach, M. S. (1991). The Effects of Board Composition and Direct Incentives on Firm Performance. Financial Management, 20(4), p.101-112. 
IBGC - Instituto Brasileiro de Governança Corporativa. (2010). Código das melhores práticas de Governança Corporativa. IBGC: São Paulo, Brasil.

Imhoff, G. (2003). Accounting Quality, Auditing and Corporate Governance. Recuperado em 31 maio, 2011 de http://ssrn.com/abstract=374380.

Instrução CVM no 308, de 14 de maio de 1999. (1999). Dispõe sobre o registro e o exercício da atividade de auditoria independente no âmbito do mercado de valores mobiliários, define os deveres e as responsabilidades dos administradores das entidades auditadas no relacionamento com os auditores independentes, e revoga as Instruções CVM nos. 216, de 29 de junho de 1994, e 275, de 12 de março de 1998. Rio de Janeiro: Comissão de Valores Mobiliários. Recuperado em 26 maio, 2011 de http:// www.cvm.gov.br/asp/cvmwww/atos/exiato.asp?File=\%5Cinst\%5Cinst308.htm.

Jensen, M, C. \& Meckling, W, H. (1976). Theory of the firm: managerial behavior, agency costs and ownership structure. Journal of Financial Economics, 3(4), p. 305-360.

La Porta, R., Lopes-de-Silanez, F., Sheifer, A. \& Vishny, R. (2000). Investor Protection and Corporate Governance. Journal of Financial Economics, 58(1-2), p. 3-27.

Lennox, C. (1999). Are large auditors more accurate than small auditors? Accounting and Business Research, 29(3), p. 217-227.

Lin, Z, J. \& Liu, M. (2009). The impact of corporate governance on auditor choice: Evidence from China. Journal of International Accounting, Auditing and Taxation, 18(1), p. 44-59.

Lopes, A. B. \& Walker, M. (2012). Firm-level incentives and the informativeness of accounting reports: an experiment in Brazil. Working Paper SSRN. Recuperado em 26 maio, 2011 de http://papers.ssrn. com/sol3/papers.cfm?abstract_id=1095781.

Pereira, A. N. (2011). Determinantes do atraso em auditoria externa (audit delay) em companhias brasileiras. Dissertação de Mestrado Profissional em Ciências Contábeis. Fundação Instituto Capixaba de Pesquisas em Contabilidade, Economia e Finanças, Vitória - ES - Brasil.

Petroni, K \& Beasley, M. (1996). Errors in Accounting Estimates and Their Relation to Audit Firm Type. Journal of Accounting Research, 34(1), p.151-171.

Shleifer, A. \& Vishny, R. W. (1997). A survey of corporate governance. The Journal of Finance, 52(2), p. 737-783.

Simunic, D. A. (1980). The Pricing of Audit Services: Theory and Evidence. Journal of Accounting Research, 18(1), p. 161-90.

Solomon, I., Shields. M. D. \& Whittington. O. R. (1999). What do industry-specialist auditors know? Journal of Accounting Research, 37(1), p.191-208.

Tirole, J. (2001). Corporate Governance. Econometric Society, 69(1), p 1-35.

Watts, L, R. \& Zimmerman, L, J. (1986). Positive Accounting Theory. Prentice-Hall.

Zingales, L. (1998). Corporate governance. New York: MacMillan. 